OPEN

SUBJECT AREAS: CONDENSED-MATTER PHYSICS

PLANETARY SCIENCE

Received 20 May 2014

Accepted

9 June 2014

Published

8 July 2014

Correspondence and requests for materials should be addressed to

G.-R.Q.

(iqianguangrui@ gmail.com)

\title{
Novel Hydrogen Hydrate Structures under Pressure
}

\author{
Guang-Rui Qian' ', Andriy O. Lyakhov', Qiang Zhu' , Artem R. Oganov ${ }^{2,3,4} \&$ Xiao Dong ${ }^{1,5}$
}

\begin{abstract}
'Department of Geosciences, Stony Brook University, Stony Brook, New York $11794-2100$, USA, 2Department of Geosciences, Center for Materials by Design, and Institute for Advanced Computational Science, State University of New York, Stony Brook, NY 1 1794-2100, ${ }^{3}$ Moscow Institute of Physics and Technology, 9 Institutskiy lane, Dolgoprudny city, Moscow Region 141700, Russia, ${ }^{4}$ School of Materials Science, Northwestern Polytechnical University, Xi'an 710072, China, ${ }^{5}$ School of Physics and MOE Key Laboratory of Weak-Light Nonlinear Photonics, Nankai University, Tianjin 300071, China.
\end{abstract}

Gas hydrates are systems of prime importance. In particular, hydrogen hydrates are potential materials of icy satellites and comets, and may be used for hydrogen storage. We explore the $\mathrm{H}_{2} \mathrm{O}-\mathrm{H}_{2}$ system at pressures in the range 0-100 GPa with ab initio variable-composition evolutionary simulations. According to our calculation and previous experiments, the $\mathrm{H}_{2} \mathrm{O}-\mathrm{H}_{2}$ system undergoes a series of transformations with pressure, and adopts the known open-network clathrate structures (sII, $\left.\mathrm{C}_{0}\right)$, dense "filled ice" structures $\left(\mathrm{C}_{1}\right.$, $\mathrm{C}_{2}$ ) and two novel hydrate phases. One of these is based on the hexagonal ice framework and has the same $\mathrm{H}_{2} \mathrm{O}: \mathrm{H}_{2}$ ratio $(2: 1)$ as the $\mathrm{C}_{0}$ phase at low pressures and similar enthalpy (we name this phase $I h-\mathrm{C}_{0}$ ). The other newly predicted hydrate phase has a $1: 2 \mathrm{H}_{2} \mathrm{O}: \mathrm{H}_{2}$ ratio and structure based on cubic ice. This phase (which we name $\mathrm{C}_{3}$ ) is predicted to be thermodynamically stable above $38 \mathrm{GPa}$ when including van der Waals interactions and zero-point vibrational energy, and explains previously mysterious experimental $\mathrm{X}$-ray diffraction and Raman measurements. This is the hydrogen-richest hydrate and this phase has a remarkable gravimetric density (18 wt.\%) of easily extractable hydrogen.

M olecular compounds (cocrystals) of water ice $\left(\mathrm{H}_{2} \mathrm{O}\right)$ and hydrogen $\left(\mathrm{H}_{2}\right)$ are known to form clathrate structures with hydrogen molecules encapsulated as guests in the host sublattice formed by water molecules. Hydrogen hydrates, as environmentally clean and efficient hydrogen storage materials, have excited significant interest. Extensive literature exists from both experimental ${ }^{1-12}$ and theoretical ${ }^{13,14}$ sides. Aside from the $\mathrm{H}_{2}$ molecules, many other small molecules can be encapsulated as guests in clathrates at elevated pressure (e.g., noble gases, nitrogen, oxygen, methane, chlorine). (See Ref. [15] and references therein) Hydrogen hydrates are important as potentially major materials of icy satellites and comets, and hydrogen storage materials.

Twenty years after the first report of the formation of two filled-ice hydrogen hydrates by Vos et al. ${ }^{1}$, four hydrogen hydrate forms are known to exist at elevated pressures. Two of the hydrogen hydrates are clathrates, denoted as clathrate structure II $(\mathrm{sII})^{3,5}$ and compound $0\left(\mathrm{C}_{0}\right)^{12}$, the other two are filled ice hydrates, compound 1 $\left(\mathrm{C}_{1}\right)$ and compound $2\left(\mathrm{C}_{2}\right)^{1,2}$. The sII clathrate hydrate was synthesized under pressures of 180 to $220 \mathrm{MPa}$ at $300 \mathrm{~K}$, and its structure was shown to contain 48 hydrogen molecules and 136 water molecules in the unit cell ${ }^{3}$. The $\mathrm{C}_{0}$ clathrate was recently found to be stable near $0.5 \mathrm{GPa}$ and to have the composition $2 \mathrm{H}_{2} \mathrm{O}: 1 \mathrm{H}_{2}$ and a trigonal quartz-like structure ${ }^{12}$. The water molecules in the $\mathrm{C}_{0}$ structure are arranged in a totally new way, different from the known ices or ice sublattices in hydrates structures. This structure has space group $P 3_{2} 21$, but this could possibly go as low as $P 3_{2}$, depending on how the hydrogens are arranged ${ }^{12}$.

At higher pressures, clathrates give way to denser structures of the filled ice type. The $C_{1}$ and $C_{2}$ phases are formed at 0.36-0.9 GPa and $\sim 2.4 \mathrm{GPa}$, respectively ${ }^{1,2,11}$. The $\mathrm{C}_{1}$ hydrate has a water host framework based on iceII and a $6: 1$ water to hydrogen ratio. $C_{2}$ has a $1: 1$ ratio of water to hydrogen and is composed of water molecules in the "cubic ice" (ice-Ic) framework and rotationally disordered hydrogen molecules ${ }^{15}$. Recent experiments ${ }^{7-10}$ indicate that the $\mathrm{C}_{2}$ hydrate undergoes a structural transformation from cubic to tetragonal phase at around 10$20 \mathrm{GPa}$, with an increasing difference in the unit cell axes, and then transforms to another high-pressure phase near $\sim 45 \mathrm{GPa}$. This high-pressure phase is maintained up to at least $80 \mathrm{GPa}$ but its structure is not fully resolved. Given the difficulties in characterization of the chemical composition and crystal structure of these hydrates, and believing that new phases are likely to exist, we decided to perform a computational search to revisit the $\mathrm{H}_{2} \mathrm{O}-\mathrm{H}_{2}$ system under pressure. 


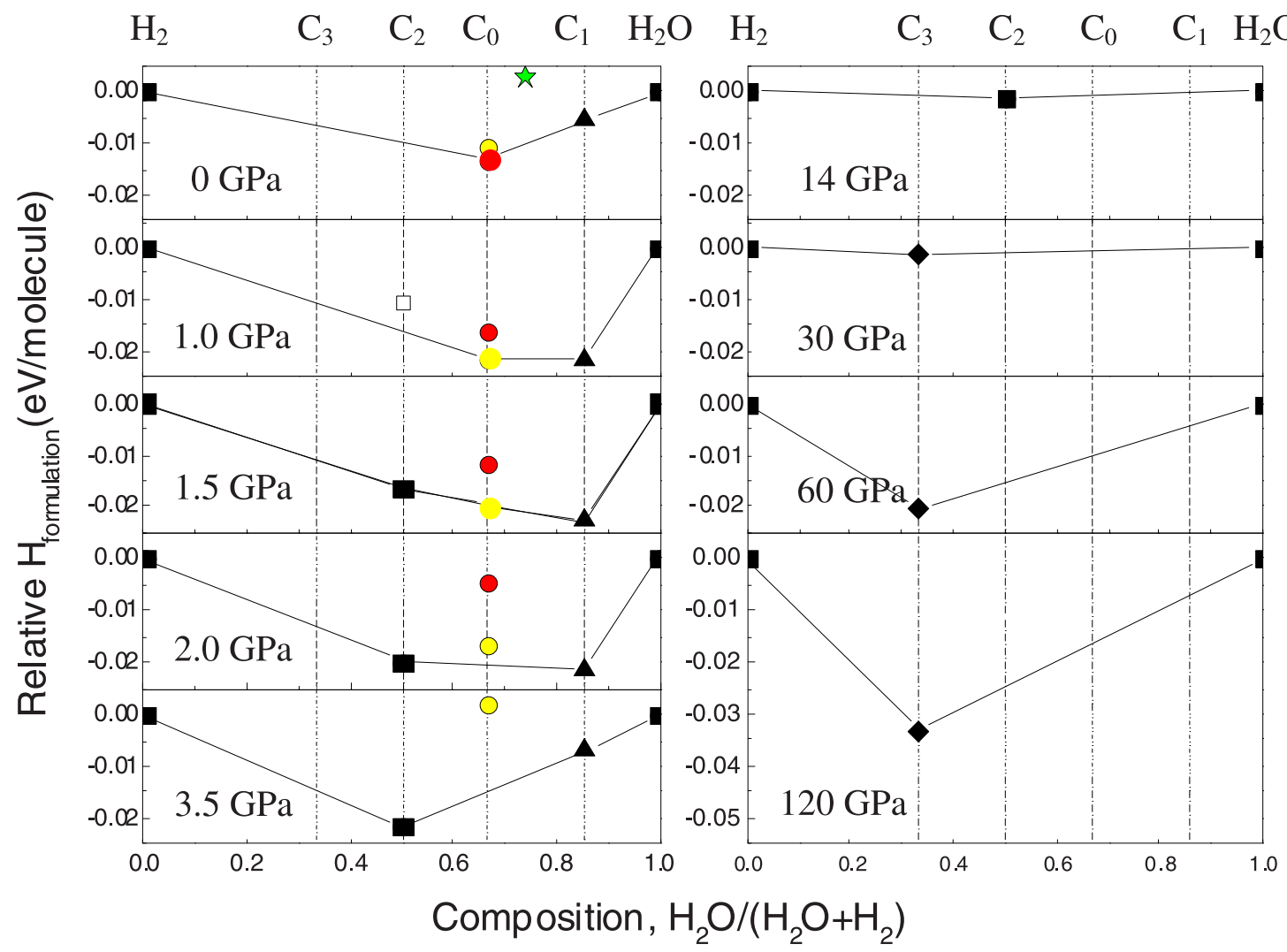

Figure 1 Convex hull diagram for $\mathrm{H}_{2} \mathrm{O}-\mathrm{H}_{2}$ system at selected pressures and zero temperature. This figure shows the enthalpy of formation (in eV/ molecule) of molecular compounds from pure $\mathrm{H}_{2} \mathrm{O}$ ice and $\mathrm{H}_{2}$. The red and yellow circles represent the $\mathrm{C}_{0}$ and $I h$ - $\mathrm{C}_{0}$ phases, respectively. The green star represents the sII structure.

\section{Results}

Using the evolutionary algorithm USPEX ${ }^{16-20}$, we explored all possible stable phases in the $\mathrm{H}_{2} \mathrm{O}-\mathrm{H}_{2}$ system (See Methods). Remarkably, we have found two novel filled ice hydrogen hydrates, and all known hydrogen hydrates (except the sII structure, because of the very large number of molecules in its unit cell). Thus, at pressures in the range $0-2 \mathrm{GPa}$, the sII structure was input separately in order to calculate stability ranges of phases in the $\mathrm{H}_{2} \mathrm{O}-\mathrm{H}_{2}$ system. Fig. 1 shows the convex hull diagram for the $\mathrm{H}_{2} \mathrm{O}-\mathrm{H}_{2}$ system.

With including the van der Waals (vdW) dispersion forces (See Methods), our results are generally in very good agreement with experiments, but with several novel aspects. At $0 \mathrm{GPa}$, the $\mathrm{C}_{0}, \mathrm{C}_{1}$ and a novel hydrogen hydrate phase are found stable or nearly stable in the $\mathrm{H}_{2} \mathrm{O}-\mathrm{H}_{2}$ system, while the sII phase is metastable $(\sim 0.013 \mathrm{eV} /$ molecule less stable than the mixture of stable compounds $\mathrm{C}_{0}$ and $\mathrm{C}_{1}$ ). The structure of the novel hydrogen hydrate is based on the framework of hexagonal ice (ice-Ih), with two hydrogen molecules hosted inside channels running along the hexagonal axis (Fig. 2a). It has a $2: 1$ ratio of water to hydrogen, same as $\mathrm{C}_{0}$, and space group $C c$. We name it $I h-\mathrm{C}_{0}$ to distinguish from $\mathrm{C}_{0}$. The enthalpy of the $I h-\mathrm{C}_{0}$ phase is close to $\mathrm{C}_{0}$, and is slightly lower at pressures above $\sim 0.4 \mathrm{GPa}$ (Fig. S2 in Supplementary Information). At $1.5 \mathrm{GPa}$, in addition to the $\mathrm{C}_{0}, I h-\mathrm{C}_{0}$ and $\mathrm{C}_{1}$ phases, the hydrate phase $\mathrm{C}_{2}$ with an ice-Ic framework structure becomes stable.

At pressures above $2 \mathrm{GPa}$, the $\mathrm{C}_{0}$ and $I h-\mathrm{C}_{0}$ phases are calculated to be above the convex hull, indicating that these phases become unstable against decomposition into $\mathrm{C}_{1}$ and $\mathrm{C}_{2}$. Above $3.5 \mathrm{GPa}$, the $\mathrm{C}_{1}$ phase will also become unstable, and the $\mathrm{C}_{2}$ phase will remain the only stable hydrate. For hydrate $\mathrm{C}_{2}$, USPEX calculations uncovered at least four typical energetically favorable candidate structures at different pressures, $P 4_{1} 2_{1} 2, I 4_{1} / a m d, P n a 2_{1}$ and $I 4_{1} m d$ (Fig. S3. in Supplementary Information). These structures differ in the orienta- (a)

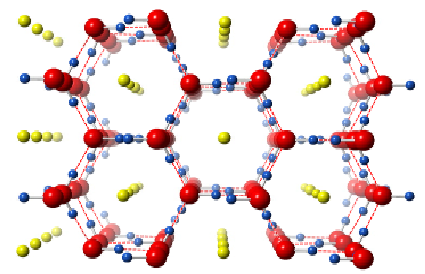

(b)

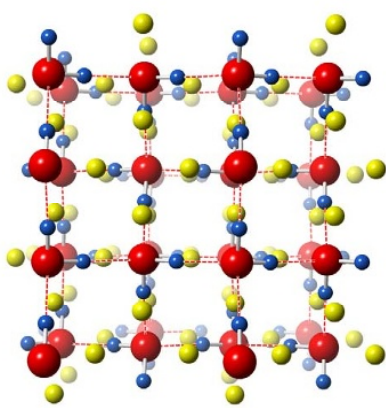

(c)

(d)
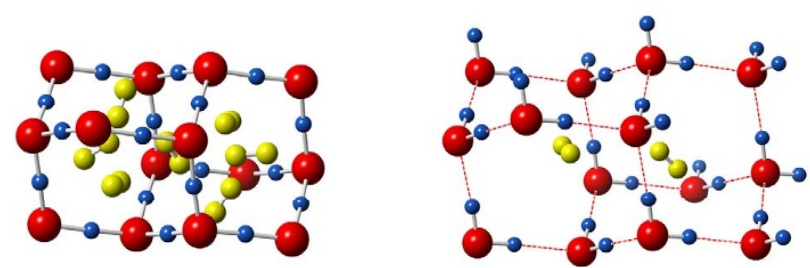

Figure $2 \mid$ Crystal structures of $I h-C_{0}$ and $C_{3}$ phases, cage structures in $C_{2}$ and $\mathrm{C}_{3}$ phases. (a) $I h-\mathrm{C}_{0}$ structure at $0.5 \mathrm{GPa}$, (b) $\mathrm{C}_{3}$ structure at $30 \mathrm{GPa}$, (c) cages formed by water molecules in $\mathrm{C}_{3}$ at $100 \mathrm{GPa}$, the hydrogen molecules are located at the center of each chair-like $\mathrm{H}-\mathrm{O}$ ring, (d) cages in "filled ice-Ic" $\mathrm{C}_{2}$, hydrogen molecules are in the center of the cage. Large red and small blue spheres are $\mathrm{O}$ and $\mathrm{H}$ atoms in water molecules, respectively; the yellow spheres represent the $\mathrm{H}_{2}$ molecules in (a) and (b), and represent $\mathrm{H}$ atoms in (c) and (d). Red dashed lines represent hydrogen bonds. 


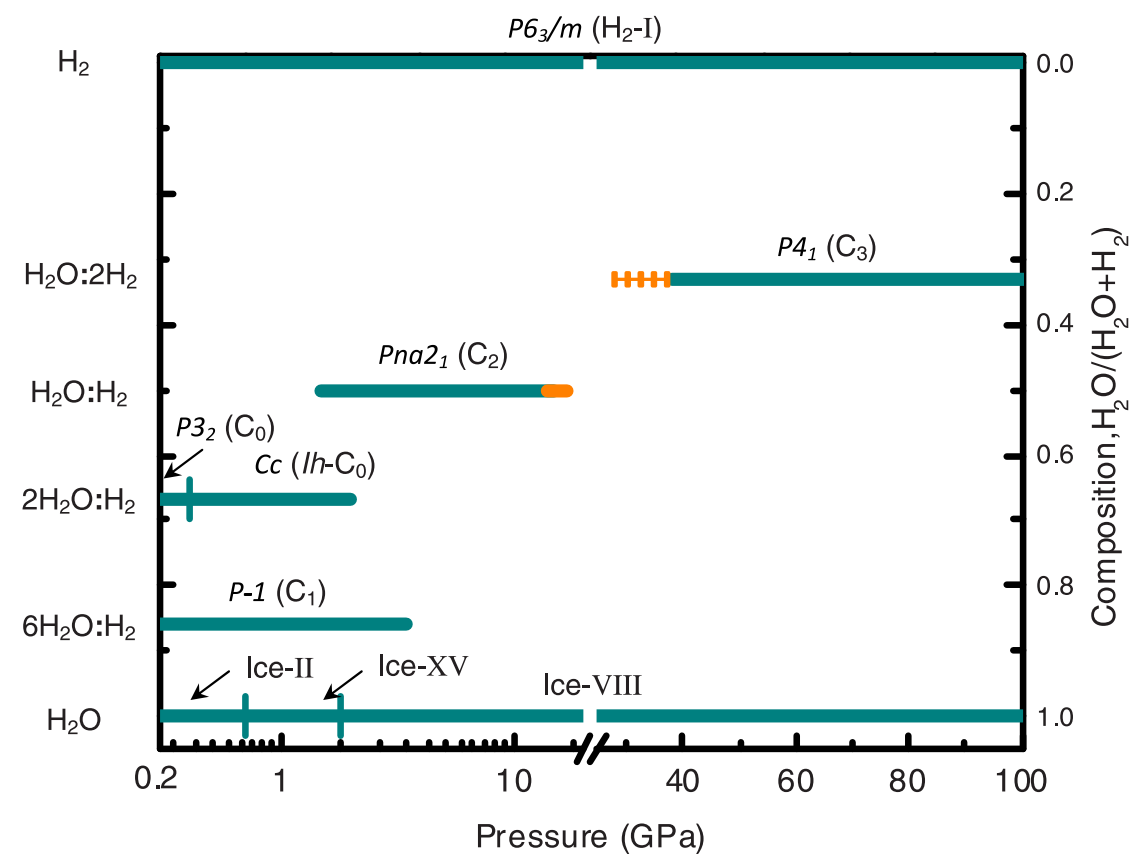

Figure 3 Phase diagram of the $\mathrm{H}_{2} \mathrm{O}-\mathrm{H}_{2}$ system. The stability ranges of $\mathrm{C}_{2}$ and $\mathrm{C}_{3}$ phases are calculated with and without $\mathrm{ZPE}$ effect. The solid orange line represents extra stability range added due to $\mathrm{ZPE}$, the dashed orange line represents regions that become unstable after inclusion of the ZPE.

tions of water and hydrogen molecules, which is similar to Ref. [15]. The $\mathrm{C}_{2}$ phase will lose stability at $\sim 14 \mathrm{GPa}$, which is much lower than $40 \mathrm{GPa}$ suggested in the previous study ${ }^{7,10}$. We explain this by metastable persistence of $\mathrm{C}_{2}$ up to the pressure of $40 \mathrm{GPa}$. Between 14-28 GPa, there are, unexpectedly, no thermodynamically stable hydrates.

Near $30 \mathrm{GPa}$, another novel $\mathrm{H}_{2} \mathrm{O}-\mathrm{H}_{2}$ phase is found to be stable at zero temperature. It has a $1: 2$ water to hydrogen ratio, and net composition $\mathrm{H}_{6} \mathrm{O}$. This novel hydrogen hydrate, which we name $\mathrm{C}_{3}$, has the highest hydrogen concentration among all hydrogen hydrates. If it can be synthesized at low pressures, it would be an attractive hydrogen storage material, having $18 \mathrm{wt} \%$ concentration of easily separable (non-water) hydrogen. The $\mathrm{C}_{3}$ structure has space group $P 4_{1}$ and is also based on the framework of ice-Ic (Fig. 2b), similar to low-pressure hydrate $\mathrm{C}_{2}$. The unit cell of $\mathrm{C}_{3}$ contains four water molecules, the $\mathrm{H}_{2}$ molecules are located at the center of chairlike $\mathrm{H}-\mathrm{O}$ rings (formed by six oxygen and six hydrogen atoms) that form faces of the cage, as shown in Fig. 2c. Differently, in the $\mathrm{C}_{2}$ hydrate, the $\mathrm{H}_{2}$ molecules are in the center of the water cages (Fig. 2d). According to our calcualtions, the $\mathrm{C}_{3}$ phase will remain stable up to at least $120 \mathrm{GPa}$.

Our theoretical calculations indicate that the $\mathrm{H}_{2} \mathrm{O}-\mathrm{H}_{2}$ system contains several stable phases, including open-network clathrate structures $\left(\mathrm{C}_{0}\right)$ and dense filled ice phases $\left(I h-\mathrm{C}_{0}, \mathrm{C}_{1}, \mathrm{C}_{2}\right.$ and $\left.\mathrm{C}_{3}\right)$. The $\mathrm{C}_{0}$ phase is predicted to be stable at pressures below $1.5 \mathrm{GPa}$, which is close to the experimental result (below $0.8 \mathrm{GPa}^{12}$ ). The $\mathrm{C}_{1}$ phase is predicted to be stable at pressures below $3.5 \mathrm{GPa}$, also close to the experimentally determined transition pressure of $2.5 \mathrm{GPa}^{1}$. The zero-point vibration energy (ZPE) significantly affects the relative stability of hydrogen-rich structures ${ }^{21}$. We have estimated the ZPE within the quasi-harmonic approximation ${ }^{22}$ to refine the stability ranges of $\mathrm{C}_{2}$ and $\mathrm{C}_{3}$ phases above $10 \mathrm{GPa}$. When considering the $\mathrm{ZPE}$, the stability field of the $\mathrm{C}_{2}$ phase expands up to $\sim 19 \mathrm{GPa}$, but this phase remains dynamically stable, and thus can exist as a metastable material at pressures of at least $60 \mathrm{GPa}$ (Fig. S9 and Fig. S10 in Supplementary Information).

The $\mathrm{C}_{3}$ phase starts to be energetically favorable above $\sim 38 \mathrm{GPa}$ when including $\mathrm{ZPE}$, as shown in Fig. 3. Thus, the novel $\mathrm{C}_{3}$ phase can be synthesized in hydrogen-rich conditions at pressures starting from $38 \mathrm{GPa}$. This theoretical value agrees well with the experimentally observed formation at 45-50 GPa of a mysterious phase of unknown composition ${ }^{7,10}$. As shown in Fig. 4, calculations of the Raman shift ${ }^{23}$ reveal differences between the $\mathrm{C}_{2}$ and $\mathrm{C}_{3}$ phases in the $\mathrm{H}_{2}-\mathrm{D}_{2} \mathrm{O}$ system. The Raman shift of $\mathrm{C}_{3}$ phase, rather than an amorphous phase, agrees very well with the lower Raman frequencies of the vibron for the hydrogen molecules observed in Ref. [9]. The black rhombi in Fig. 4 indicate that some of the $\mathrm{H}_{2}-\mathrm{D}_{2} \mathrm{O} \mathrm{C}_{3}$ sample encountered decomposition when quenched to low pressure. The variation of lattice parameters of the ice host structure in hydrates with pressure, revealed by our theoretical calculations, also agrees well with experimental XRD results ${ }^{10}$. At $55 \mathrm{GPa}$, our calculation

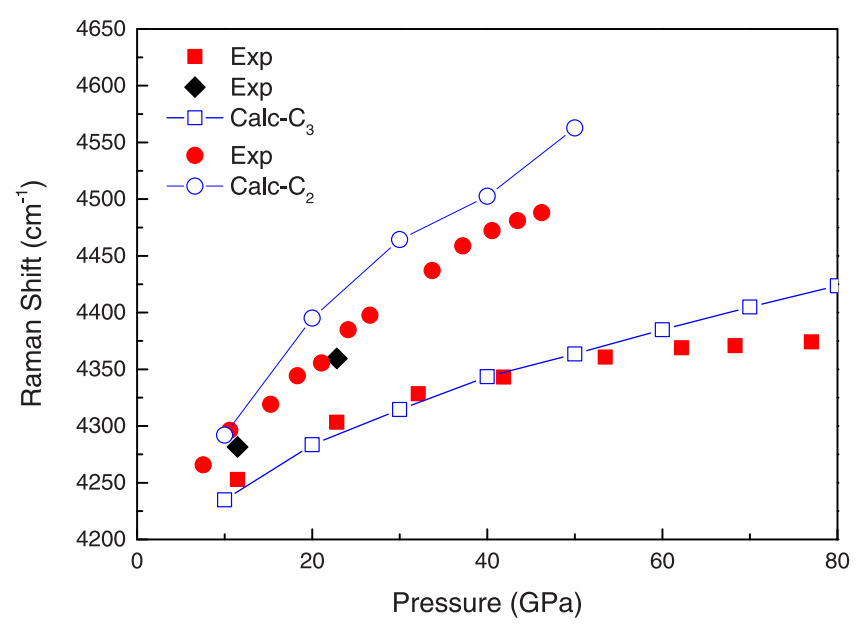

Figure $4 \mid$ Variations of the Raman shift of the vibron for the $\mathrm{H}_{2}$ molecules with pressure from experimental data in Ref. [9] and our theoretical calculations. The red and black symbols are the experimental data for $\mathrm{H}_{2}$ vibrons in the $\mathrm{H}_{2}-\mathrm{D}_{2} \mathrm{O}$ sample. The blue open circles and squares indicate the Raman shift calculation for $\mathrm{C}_{2}$ and $\mathrm{C}_{3}$ phases of $\mathrm{H}_{2}-$ $\mathrm{D}_{2} \mathrm{O}$ system, respectively. 


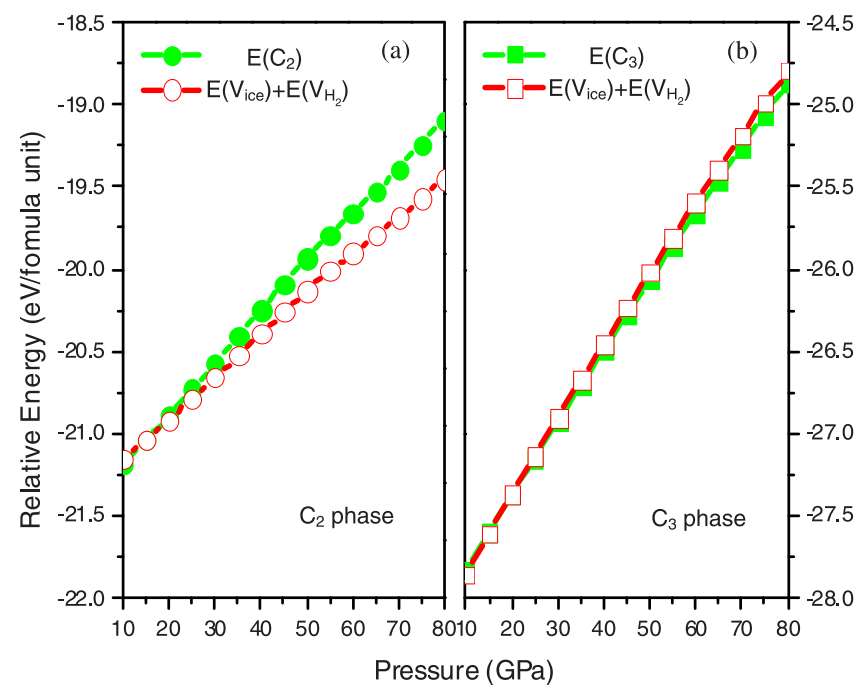

Figure 5 Internal energy of the $\mathrm{C}_{2}$ and $\mathrm{C}_{3}$ phases relative to the isochoric mixture of $\mathrm{H}_{2} \mathrm{O}$ and $\mathrm{H}_{2}$. Green lines represent the energy of the hydrate phases; red lines represent the energy of the isochoric mixture of ice-VIII and $\mathrm{H}_{2}$-I phases.

gives lattice parameter of $C_{3}$ phase $a=b=4.00 \AA$ and $c=5.67 \AA$, corresponding to cubic ice sublattice with periodicity $5.67 \AA$, whereas experiment gives $\sim 5.5 \AA^{10}$.

At low pressure, the $\mathrm{C}_{2}$ adopts a "cubic ice" host structure and then transforms to a "tetragonal" one at $\sim 20 \mathrm{GPa}^{10}$ (Fig. S4 in Supplementary Information). When forming the $C_{3}$ phase at increased pressure and in excess of $\mathrm{H}_{2}$, the ice host structure transforms to the "cubic ice" again. The change from tetragonal to "cubic" structure occurs before $\mathrm{H}$-bond symmetrization transition happens in "tetragonal" type $\mathrm{C}_{2}$ at $\sim 55 \mathrm{GPa}$. Thus, such structural transformation is unrelated to symmetrization of the $\mathrm{H}$-bonds, but comes from the emergence of the $\mathrm{C}_{3}$ phase. For the hydrate $\mathrm{C}_{3}$, the $\mathrm{H}$-bond symmetrization is predicted to occur at $\sim 120 \mathrm{GPa}$ (Fig. S5 in Supplementary Information), which is close the theoretical H-bond symmetrization pressure in ice-VII ${ }^{14}$.

\section{Discussion}

The $\mathrm{C}_{2}$ and $\mathrm{C}_{3}$ hydrates have a similar ice host framework, but the different numbers of hydrogen molecules and their different locations and orientations bring huge differences in phase stability range. In the $C_{2}$ phase, hydrogen molecules stay in the centers of cages formed by water molecules in contrast to $\mathrm{C}_{3}$ phase, where they are located at the faces of the cages. To clarify the causes of stability of hydrogen hydrates, we used Bader analysis ${ }^{24,25}$, and focused on the $\mathrm{C}_{2}$ and $\mathrm{C}_{3}$ phases (Fig. S6 in Supplementary Information). We found a very small charge transferred from $\mathrm{H}_{2}$ to water molecules, so that the $\mathrm{H}_{2}$ molecules are slightly positively charged, and $\mathrm{H}_{2} \mathrm{O}$ molecules carry a slight negative charge. The magnitudes of these charges are $\sim 10^{-3}-10^{-2}$ per molecule. This suggests that interactions between these molecules are almost purely steric, mainly related to packing density and shapes of the molecules. Comparing Bader volumes of the $\mathrm{H}_{2} \mathrm{O}$ and $\mathrm{H}_{2}$ molecules in the hydrates and in pure $\mathrm{H}_{2} \mathrm{O}$ and $\mathrm{H}_{2}$, we see that water molecules occupy slightly larger volume in the hydrates, whereas hydrogen molecules occupy much less space in $\mathrm{C}_{3}$ hydrate than in pure $\mathrm{H}_{2}$ - this leads to net densification, stabilizing this phase in a wide pressure range. For the $\mathrm{C}_{2}$ hydrate, the $\mathrm{H}_{2}$ molecules have lower volume than in pure $\mathrm{H}_{2}$ only at pressures below $\sim 10 \mathrm{GPa}$, which explains its instability at higher pressures.

Having considered the $P V$-term in the enthalpy $(H=E+P V)$, to get additional insight, we turned to the internal energy $E$ and its changes when the $\mathrm{H}_{2}$ and $\mathrm{H}_{2} \mathrm{O}$ molecules are placed from the hydrate into pure $\mathrm{H}_{2}$ and $\mathrm{H}_{2} \mathrm{O}$ phases, while keeping molecular volumes fixed at their values in the hydrate (Fig. 5). This energy characterizes the net balance of the vdW attraction and steric repulsion between the molecules: this net effect is very small in the $\mathrm{C}_{3}$ phase (slightly destabilizing below $\sim 30 \mathrm{GPa}$ and slightly stabilizing above $\sim 30 \mathrm{GPa}$ ). The remarkably wide stability field of the $\mathrm{C}_{3}$ phase is therefore mostly due to its high density and only to a small extent to more favorable intermolecular interactions. A much more interesting picture is observed for the $\mathrm{C}_{2}$ phase (Fig. 5a): we find its slight energetic stabilization below $\sim 15 \mathrm{GPa}$, and an increasingly large destabilization at higher pressures. This explains why $\mathrm{C}_{2}$ is unstable at high pressures, and furthermore, it is clear that the increasing energetic instability of the $\mathrm{C}_{2}$ phase is responsible for the displacive phase transition, metastably occurring on overcompression and transforming the cubic $\mathrm{H}_{2} \mathrm{O}$ host sublattice into tetragonal, to enable better packing of the molecules.

Our calculations found that a $\mathrm{C}_{3}$-type phase is stable in the $\mathrm{H}_{2} \mathrm{O}-$ He system at 8-75 GPa (without including zero-point energy), and this phase is denser than the mixture of $\mathrm{H}_{2} \mathrm{O}$ and $\mathrm{He}$. On the other hand, no such phase was found in the $\mathrm{H}_{2} \mathrm{O}-\mathrm{Ne}$ system, and indeed the $\mathrm{C}_{3}$ phase is not packing-efficient in this system (Fig. S7 and Fig. S8 in Supplementary Information). He and $\mathrm{Ne}$ are both chemically extremely inert, their almost only differences are size and (here insignificant) mass. Stability of $\mathrm{He}-\mathrm{C}_{3}$ and instability of $\mathrm{Ne}-\mathrm{C}_{3}$ hydrates reinforce our conclusion made for the $\mathrm{H}_{2} \mathrm{O}-\mathrm{H}_{2}$ system, that stability of this novel phase comes not from specific bonding interactions between the molecules, and not even their shapes, but is mostly due to their very efficient packing.

In summary, using the evolutionary algorithm USPEX, we explored the $\mathrm{H}_{2} \mathrm{O}-\mathrm{H}_{2}$ system at pressures of up to $100 \mathrm{GPa}$. Stoichiometries and stability fields of $\mathrm{H}_{2} \mathrm{O}-\mathrm{H}_{2}$ hydrate phases have been studied. A series of pressure-induced transformations found by theory closely coincides with experimental data, but also new insight was obtained. A novel $I h-\mathrm{C}_{0}$ structure is predicted to have a very close enthalpy to the recently discovered $\mathrm{C}_{0}$ structure. At pressures above $38 \mathrm{GPa}$, novel hydrogen hydrate $\mathrm{C}_{3}$, based on cubic ice Ic, is predicted to be stable. With stoichiometry $\mathrm{H}_{2} \mathrm{O}: 2 \mathrm{H}_{2}$, this is the hydrogen-richest hydrate known to date. With gravimetric density of easily removable hydrogen (18 wt.\%), this is a promising hydrogen storage material that can find practical applications if its synthesis pressure can be decreased.

\section{Methods}

Crystal structure prediction. Predictions were done using the USPEX code in its variable-composition mode at several pressures $(0,1,2,5,10,20,50$ and $100 \mathrm{GPa})$ and zero temperature. A number of studies illustrate the power of the USPEX method $^{26-28}$. We have done two types of variable-composition structure predictions in searching for all stable phases in the $\mathrm{H}-\mathrm{O}$ system: (1) In the $\mathrm{H}-\mathrm{O}$ system, assembling the structures from atoms, and (2) In the $\mathrm{H}_{2} \mathrm{O}-\mathrm{H}_{2}$ system with giving $\mathrm{H}_{2} \mathrm{O}$ and $\mathrm{H}_{2}$ molecules as structure building blocks. We have found that, even in the $\mathrm{H}-\mathrm{O}$ system, all low-enthalpy states at pressures of our interest $(<120 \mathrm{GPa})$ are actually made of well-defined $\mathrm{H}_{2} \mathrm{O}$ and $\mathrm{H}_{2}$ molecules. This allowed us to focus on molecular-type calculations, capable of efficiently dealing with large systems, without loss of rigor.

Given molecular nature of all stable and nearly stable compounds in this system, we searched for the packing of well-defined $\mathrm{H}_{2} \mathrm{O}$ and $\mathrm{H}_{2}$ molecules (rather than $\mathrm{H}$ and $\mathrm{O}$ atoms), by applying the specially designed constrained global optimization algorithm, considering structures with up to 24 molecules (i.e. up to 72 atoms) per primitive unit cell.

DFT calculations. Structure relaxations were done using density functional theory (DFT) within van der Waals (vdW) functional optB88-vdW ${ }^{29}$ in the framework of the all-electron projector augmented wave $(\mathrm{PAW})^{30}$ method as implemented in the VASP $^{31}$ code. The plane wave kinetic energy cutoff of $600 \mathrm{eV}$ and Gamma-centered $k$-point meshes with the reciprocal space resolution of $2 \pi \times 0.05 \AA$ were used. Having identified the most stable compositions and candidate structures, we relaxed them at pressures from $1 \mathrm{~atm}$ to $120 \mathrm{GPa}$ with an even higher cutoff of $800 \mathrm{eV}$ to refine their thermodynamic properties and stability fields. Structure relaxations proceeded until net forces on atoms were below $1 \mathrm{meV} / \AA$, which gave us enthalpies converged to better than $1 \mathrm{meV} /$ atom.

It is expected that the relative contribution of hydrogen bonding (H-bonding) and van der Waals (vdW) dispersion forces has a significant impact on the phase transition pressures and cohesive properties of the various crystalline ice phases ${ }^{32}$. This is 
also confirmed by our calculations for the phase transition pressures of ice phases from optB88-vdW, GGA ${ }^{33}$ calculations and experiments (Fig. S1 in Supplemental Information). Thus, all calculations included the vdW functional to treat the $\mathrm{vdW}$ forces, unless stated otherwise.

1. Vos, W. L., Finger, L. W., Hemley, R. J. \& Mao, H.-K. Novel $\mathrm{H}_{2}-\mathrm{H}_{2} \mathrm{O}$ clathrates at high pressures. Phys. Rev. Lett. 71, 3150-3153 (1993).

2. Vos, W. L., Finger, L. W., Hemley, R. J. \& kwang Mao, H. Pressure dependence of hydrogen bonding in a novel $\mathrm{H}_{2} \mathrm{O}-\mathrm{H}_{2}$ clathrate. Chem. Phys. Lett. 257, 524-530 (1996).

3. Mao, W. L. et al. Hydrogen clusters in clathrate hydrate. Science 297, 2247-2249 (2002).

4. Mao, W. L. \& Mao, H.-K. Hydrogen storage in molecular compounds. Proc. Natl. Acad. Sci. USA 101, 708-710 (2004).

5. Lokshin, K. A. et al. Structure and dynamics of hydrogen molecules in the novel clathrate hydrate by high pressure neutron diffraction. Phys. Rev. Lett. 93, 125503 (2004).

6. Hirai, H., Ohno, S., Kawamura, T., Yamamoto, Y. \& Yagi, T. Changes in vibration modes of hydrogen and water molecules and in lattice parameters with pressure for filled-ice hydrogen hydrates. J. Phys. Chem. C 111, 312-315 (2007).

7. Machida, S.-I., Hirai, H., Kawamura, T., Yamamoto, Y. \& Yagi, T. Structural changes of filled ice ic structure for hydrogen hydrate under high pressure. J. Chem. Phys. 129, 224505 (2008).

8. Machida, S.-I., Hirai, H., Kawamura, T., Yamamoto, Y. \& Yagi, T. Structural changes and intermolecular interactions of filled ice ic structure for hydrogen hydrate under high pressure. J. Phys.: Conf. Ser. 215, 012060 (2010).

9. Machida, S.-I., Hirai, H., Kawamura, T., Yamamoto, Y. \& Yagi, T. Isotopic effect and amorphization of deuterated hydrogen hydrate under high pressure. Phys. Rev. B 83, 144101 (2011).

10. Hirai, H. et al. Structural changes of filled ice ic hydrogen hydrate under low temperatures and high pressures from 5 to 50 GPa. J. Chem. Phys. 137, 074505 (2012)

11. Strobel, T. A., Somayazulu, M. \& Hemley, R. J. Phase behavior of $\mathrm{H}_{2}+\mathrm{H}_{2} \mathrm{O}$ at high pressures and low temperatures. J. Phys. Chem. C 115, 4898-4903 (2011).

12. Efimchenko, V. et al. New phase in the water-hydrogen system. J. Alloy. Comp. 509, Supplement 2, S860-S863 (2011).

13. Lenz, A. \& Ojamäe, L. Structures of the I-, II- and H-methane clathrates and the Icemethane clathrate phase transition from quantum-chemical modeling with force-field thermal corrections. J. Phys. Chem. A 115, 6169-6176 (2011).

14. Zhang, J., Kuo, J.-L. \& Iitaka, T. First principles molecular dynamics study of filled ice hydrogen hydrate. J. Chem. Phys. 137, 084505 (2012).

15. Loveday, J. S. \& Nelmes, R. J. High-pressure gas hydrates. Phys. Chem. Chem. Phys. 10, 937-950 (2008).

16. Oganov, A. R. \& Glass, C. W. Crystal structure prediction using ab initio evolutionary techniques: Principles and applications. J. Chem. Phys. 124, - (2006).

17. Oganov, A. R., Ma, Y., Lyakhov, A. O., Valle, M. \& Gatti, C. Evolutionary crystal structure prediction as a method for the discovery of minerals and materials. Rev. Mineral. Geochem. 71, 271-298 (2010).

18. Oganov, A. R., Lyakhov, A. O. \& Valle, M. How evolutionary crystal structure prediction works-and why. Acc. Chem. Res. 44, 227-237 (2011).

19. Zhu, Q., Oganov, A. R., Glass, C. W. \& Stokes, H. T. Constrained evolutionary algorithm for structure prediction of molecular crystals: methodology and applications. Acta Crystallogr., Sect. B: Struct. Sci 68, 215-226 (2012).

20. Zhu, Q. et al. Stability of xenon oxides at high pressures. Nat. Chem. 5, 61-65 (2012)

21. Pickard, C. J. \& Needs, R. J. Structure of phase III of solid hydrogen. Nat. Phys. 3, $473-476$ (2007).
22. Togo, A., Oba, F. \& Tanaka, I. First-principles calculations of the ferroelastic transition between rutile-type and $\mathrm{CaCl}_{2}$-type $\mathrm{SiO}_{2}$ at high pressures. Phys. Rev. B 78, 134106 (2008).

23. Lazzeri, M. \& Mauri, F. First-principles calculation of vibrational raman spectra in large systems: Signature of small rings in crystalline $\mathrm{SiO}_{2}$. Phys. Rev. Lett. 90, 036401 (2003).

24. Bader, R. F. W. Atoms in Molecules - A Quantum Theory (Oxford University Press, 1990).

25. Henkelman, G., Arnaldsson, A. \& Jonsson, H. A fast and robust algorithm for bader decomposition of charge density. Comput. Mater. Sci. 36, 354-360 (2006).

26. Hu, C.-H. et al. Pressure-induced stabilization and insulator-superconductor transition of bh. Phys. Rev. Lett. 110, 165504 (2013).

27. Zhu, Q., Oganov, A. R. \& Lyakhov, A. O. Novel stable compounds in the Mg-O system under high pressure. Phys. Chem. Chem. Phys. 15, 7696-7700 (2013).

28. Zhang, W. et al. Unexpected stable stoichiometries of sodium chlorides. Science 342, 1502-1505 (2013)

29. Klimeš, J., Bowler, D. R. \& Michaelides, A. Van der waals density functionals applied to solids. Phys. Rev. B 83, 195131 (2011).

30. Blöchl, P. E. Projector augmented-wave method. Phys. Rev. B 50, 17953-17979 (1994).

31. Kresse, G. \& Furthmüller, J. Efficient iterative schemes for $a b$ initio total-energy calculations using a plane-wave basis set. Phys. Rev. B 54, 11169-11186 (1996).

32. Santra, B. et al. Hydrogen bonds and van der waals forces in ice at ambient and high pressures. Phys. Rev. Lett. 107, 185701 (2011).

33. Perdew, J. P., Burke, K. \& Ernzerhof, M. Generalized gradient approximation made simple. Phys. Rev. Lett. 77, 3865-3868 (1996).

\section{Acknowledgments}

We thank the National Science Foundation (EAR-1114313, DMR-1231586), DARPA (Grants No. W31P4Q1210008 and No. W31P4Q1310005) and the Government (No. 14.A12.31.0003) of Russian Federation (Project No. 8512) for financial support, and Foreign Talents Introduction and Academic Exchange Program (No. B08040). We also thank Purdue University Teragrid and TACC Stampede system for providing computational resources and technical support for this work (Charge No.: TG-DMR110058)

\section{Author contributions}

G.R.Q., A.O.L., Q.Z., A.R.O. and X.D. designed research, performed simulations, analyzed data, and wrote the manuscript.

\section{Additional information}

Supplementary information accompanies this paper at http://www.nature.com/ scientificreports

Competing financial interests: The authors declare no competing financial interests. How to cite this article: Qian, G.-R., Lyakhov, A.O., Zhu, Q., Oganov, A.R. \& Dong, X Novel Hydrogen Hydrate Structures under Pressure. Sci. Rep. 4, 5606; DOI:10.1038/ srep05606 (2014)

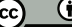

This work is licensed under a Creative Commons Attribution 4.0 International License. The images or other third party material in this article are included in the article's Creative Commons license, unless indicated otherwise in the credit line; if the material is not included under the Creative Commons license, users will need to obtain permission from the license holder in order to reproduce the material. To view a copy of this license, visit http://creativecommons.org/licenses/by/4.0/ 